\title{
Relationship between lower urinary tract symptoms and inguinal hernia
}

\author{
Aykut Buğra Sentürk ${ }^{1}$, Musa Ekici ${ }^{1}$, Ibrahim Tayfun Sahiner ${ }^{2}$, Tuncay Tas ${ }^{3}$, Basri Cakiroglu ${ }^{4}$ \\ ${ }^{1}$ Hitit University Corum Training and Research Hospital, Urology Department, Turkey; \\ ${ }^{2}$ Hitit University Corum Training and Research Hospital, General Surgery Department, Turkey; \\ ${ }^{3}$ Istanbul Esenyurt University, Private Esencan Hospital, Department of Urology, Turkey; \\ ${ }^{4}$ Hisar Intercontinental Hospital, Department of Urology, Turkey.
}

\begin{abstract}
Summary Aim: To determine the relationship between inguinal hernia (and inguinal hernia subtypes) and low urinary tract symptoms (LUTS) due to benign prostate hyperplasia (BPH), that could be more common than we think.

Method: The study was designed retrospectively and was done in accordance with the principles of the Declaration of Helsinki, including 100 patients aged > 50 years that were divided into 2 groups: patients with $\mathrm{BPH}$ (BPH group) and patients with $\mathrm{BPH}$ and inguinal hernia (BPH-IH group 2). In addition, the BPH-IH group was subdivided according to 2 inguinal hernia subtypes; patients of BPH-IH subgroup A had direct inguinal hernia $(n=25)$ and those of BPH-IH subgroup $B$ had indirect inguinal hernia $(n=25)$.

Results: There was no statistical relationship and difference in rates between IPSS scores in both groups $(p=0.659)$ and there wasn't a significant correlation between IPSS symptom severity and type of hernia, based on chi square analysis $(p=0.104)$

Conclusion: We were not able to prove our hypothesis that patients with inguinal hernia and $\mathrm{BPH}$ would have higher IPSS scores because of voiding dysfunction.
\end{abstract}

KEY WORDS: Inguinal hernia; Lower urinary tract symptoms; Benign prostatic hyperplasia.

Submitted 19 May 2016; Accepted 19 August 2016

\section{INTRODUCTION}

Lower urinary tract symptoms (LUTS) due to benign prostatic hyperplasia (BPH) are very common among elderly men. Conditions that occur more frequently with age such as atherosclerosis, obesity, and insulin resistance, can be important underlying etiological factors for benign prostatic enlargement (BPE) that is generally caused by the histopathological condition known as $\mathrm{BPH}$. BPE, like BPH, is also commonly found among elderly men. BPE is the most common cause of LUTS in elderly men, occurring in $40 \%$ of men aged 50 years and in $90 \%$ of those aged $90-99$ years (1). In urological practice, symptom scores are used to evaluate LUTS due to $\mathrm{BPH}$. The most commonly used prostate symptom questionnaire is that which generates the International Prostate Symptom Score (IPSS), which classifies the severity of symptoms as mild (score: 0-7), moderate (score: 8-19), or severe (score: 20-35).
The prevalence of inguinal hernia increases with age, and inguinal hernias account for $75 \%$ of all abdominal hernias. It is difficult to determine the precise prevalence of inguinal hernias within the general community, however, they do occur more commonly in men with a lifetime risk of $27 \%$ (2). Multiple etiological factors are associated with hernia. Chronic cough, chronic obstructive pulmonary disease, constipation, prostatism, pregnancy, ascites, and heavy lifting are known to cause inguinal hernia, via an increase in intra-abdominal pressure. Congenital diseases of the connective tissue, collagen synthesis defects, and a family history of hernia are all considered congenital causes. Moreover, inguinal hernia is more common in smokers than in nonsmokers. It has been reported that mechanisms underlying the collagen synthesis degradation pathways could cause inguinal hernia. Overall, although inguinal hernia is one of the most common surgically treated conditions, it remains to be fully understood, given not only the multiple factors associated with its etiology and recurrence, but also the wide range of treatment alternatives available $(2,3)$.

In urology practice, the coexistence of inguinal hernia and LUTS due to BPH is very common. Patients with inguinal hernia reportedly have higher IPSSs than those without inguinal hernia (4). The aim of the present study is to determine the relationship between inguinal hernia (and inguinal hernia subtypes) and LUTS, on account of BPH.

\section{Materials AND METHOdS}

This retrospective study was undertaken in accordance with the principles of the Declaration of Helsinki, and involved 100 patients aged $>50$ years who were divided into two groups-namely, patients with BPH ("the BPH group"; $\mathrm{n}=50$ ) and patients with $\mathrm{BPH}$ and inguinal hernia ("the BPH-IH group"; $\mathrm{n}=50$ ). In addition, the BPH-IH group was subdivided according the two inguinal hernia subtypes: those in $\mathrm{BPH}-\mathrm{IH}$ subgroup $\mathrm{A}$ had a direct inguinal hernia $(\mathrm{n}=25)$, and those in BPH-IH subgroup $\mathrm{B}$ had an indirect inguinal hernia $(\mathrm{n}=25)$. Diagnosis and subtyping of inguinal hernias were performed by a general surgeon. Patients with a history of inguinal hernia repair or of prostate surgery, BPH medication use, prostate cancer, urinary tract infection, or urethral stricture disease were excluded from this study. 
IPSS and a questionnaire used to measure the quality of life according to urinary symptoms (QI) were completed by the patients in the outpatient clinic prior to physical examination. Total prostate-specific antigen (T-PSA), urine analysis, and urine culture tests were performed. IPSS was used to assess the severity of the symptoms, and the duration of the symptoms was noted. Prostate volume (anteroposterior diameter $\times$ transverse diameter $\times$ longitudinal diameter $\times 0.52$ ) was measured by suprapubic sonography using a 3.1-MHz superficial ultrasonic probe, and this was immediately followed by uroflowmetry (MMS, Holland).

\section{Statistical analysis}

Statistical analysis was performed using IBM SPSS Statistics for Windows v. 22 (IBM Corp., Armonk, NY). Descriptive statistics are presented as mean \pm standard deviation (SD) for continuous variables with normal distribution, as median \pm SD for continuous variables not normally distributed, and as number (n) and ratio for categorical variables. Data normally distributed were analyzed through the use of Shapiro-Wilk and Kolmogorov-Smirnov tests. Comparisons of two independent continuous variables were performed using the independent samples t-test and Mann-Whitney U test for normally distributed and not normally distributed data, respectively. The relationship between two categorical variables and two ratios was determined via the chisquare test. The level of statistical significance was set at $\mathrm{p}<0.05$.

\section{ResULTS}

In the total study population, the mean age was $61.45 \pm$ 8.33 years, the mean IPSS was $15.24 \pm 8.19$, the mean peak flow rate was $13.40 \pm 5.28 \mathrm{ml} / \mathrm{sec}$, and the mean prostate volume was $44.30 \pm 13.96 \mathrm{ml}$ (Table 1 ).

\section{Table 1.}

Descriptive statistics.

\begin{tabular}{|lcccc|}
\hline & $\mathbf{N}$ & Mean \pm SD & Median & Range \\
\hline Age (years) & 100 & $61.45 \pm 8.33$ & 60.5 & $45-87$ \\
\hline IPSS score & 100 & $15.24 \pm 8.19$ & 15 & $1-32$ \\
\hline $\mathbf{Q}_{\max }(\mathrm{ml} / \mathrm{sec})$ & 100 & $13.40 \pm 5.28$ & 13 & $3-32$ \\
\hline Prostate volume $(\mathrm{ml})$ & 100 & $44.30 \pm 13.96$ & 45 & $15-75$ \\
\hline
\end{tabular}

The mean age was $61.38 \pm 9.19$ years in the $\mathrm{BPH}-\mathrm{IH}$ group, versus $61.52 \pm 7.47$ years in the $\mathrm{BPH}$ group; the difference was not significant $(p=0.934)$. The mean flow rate $\left(\mathrm{Q}_{\max }\right)$ was $13.78 \pm 4.28 \mathrm{ml} / \mathrm{sec}$ (median: 15 ; range: 6-20) in the BPH-IH group, versus $13.04 \pm 6.12 \mathrm{ml} / \mathrm{sec}$ (median: 12; range: 3-32) in the BPH group; again, the difference was not significant $(p=0.175)$. The mean prostate volume in the BPH-IH group was 47.16 $\pm 13.57 \mathrm{ml}$ (median: 45; range: 15-72), versus $41.39 \pm 13.88 \mathrm{ml}$ (median: 40; range: $20-75)$ in the BPH group; this difference was significant ( $\mathrm{p}=0.036)$. The mean IPSS in the BPH-IH group was $14.46 \pm 8.64$ (median: 13; range: 1-32), versus $16.02 \pm$ 7.72 (median: 15.50; range: 5-30) in the $\mathrm{BPH}$ group; the difference was not significant $(p=0.348)$ (Table 2$)$.
Table 2.

Between-group comparisons.

\begin{tabular}{|c|c|c|c|c|c|c|}
\hline & Group & $\mathbf{n}$ & Mean \pm SD & Median & Range & $\mathbf{P}$ \\
\hline \multirow[t]{2}{*}{ Age (years) } & BPH-IH & 50 & $61.38 \pm 9.19$ & 62.5 & $50-84$ & 0.934 \\
\hline & BPH & 50 & $61.52 \pm 7.47$ & 60 & $45-87$ & \\
\hline \multirow[t]{2}{*}{ IPSS score } & BPH-IH & 50 & $14.46 \pm 8.64$ & 13 & $1-32$ & 0.348 \\
\hline & BPH & 50 & $16.02 \pm 7.72$ & 15.50 & $5-30$ & \\
\hline \multirow[t]{2}{*}{$\overline{\mathbf{Q}_{\max }(\mathrm{ml} / \mathrm{sec})}$} & BPH-IH & 50 & $13.78 \pm 4.28$ & 15 & $6-20$ & 0.175 \\
\hline & BPH & 50 & $13.04 \pm 6.12$ & 12 & 3-32 & \\
\hline \multirow[t]{2}{*}{ Prostate volume (ml) } & BPH-IH & 50 & $47.16 \pm 13.57$ & 45 & $15-72$ & $0.036^{*}$ \\
\hline & BPH & 50 & $41.39 \pm 13.88$ & 40 & $20-75$ & \\
\hline
\end{tabular}

Mild, moderate, and severe LUTS was observed in 10 (20\%), $22(44 \%)$, and $18(36 \%)$ of the patients in the BPH group, respectively, versus 10 (20\%), 26 (52\%), and $14(28 \%)$ of those in the BPH-IH group. There was no statistical relationship between, or difference in, the rates between the IPSSs of the two main groups $(\mathrm{p}=0.659)$ (Table 3).

Table 3.

The frequency and percentage of IPSS symptom severity, based on the chi-square test.

\begin{tabular}{|lcccc|}
\hline Group & $\begin{array}{c}\text { Mild } \\
\mathbf{n}\end{array}$ & $\begin{array}{c}\text { Moderate } \\
\mathbf{n}\end{array}$ & $\begin{array}{c}\text { Severe } \\
\mathbf{n}\end{array}$ & $\mathbf{P}$ \\
\hline BPH & $10(20 \%)$ & $22(44 \%)$ & $18(36 \%)$ & 0.659 \\
\hline BPH-IH & $10(20 \%)$ & $26(52 \%)$ & $14(28 \%)$ & 0.659 \\
\hline
\end{tabular}

Mild, moderate, and severe LUTS were noted in 8 (32\%), $9(36 \%)$, and $8(32 \%)$ of the patients in BPH-IH subgroup A (direct inguinal hernia) and in $3(12 \%), 16$ (64\%), and 6 (24\%) of those in BPH-IH subgroup B (indirect inguinal hernia). There was no significant correlation between IPSS symptom severity and hernia type, based on chi square analysis $(\mathrm{p}=0.104)($ Table 4$)$.

\section{Table 4.}

The frequency and percent of IPSS symptom severity in the hernia subgroups, based on the chi-square test.

\begin{tabular}{|lccccc|}
\hline & 0-7 Mild & 8-19 Moderate & 20+ Severe & Total & P \\
\hline Direct hernia & $8(32 \%)$ & $9(36 \%)$ & $8(32 \%)$ & $25(100 \%)$ & 0.104 \\
\hline Indirect hernia & $3(12 \%)$ & $16(64 \%)$ & $6(24 \%)$ & $25(100 \%)$ & \\
\hline Total & $11(22 \%)$ & $25(50 \%)$ & $14(28 \%)$ & $50(100 \%)$ & \\
\hline
\end{tabular}

The mean IPSS did not differ significantly between BPHIH subgroup A and subgroup B ( $\mathrm{p}=0.763)$ (Table 5$)$.

\section{Table 5.}

IPSS score in BHP-IH subgroup A and subgroup B.

\begin{tabular}{|lccccc|}
\hline & Group & $\mathbf{n}$ & Mean \pm SD & Median & P \\
\hline IPSS & BHP-IH subgroup A & 25 & $14.28 \pm 9.64$ & 13 & 0.763 \\
\hline & BHP-IH subgroup B & 25 & $14.64 \pm 7.71$ & 13 & \\
\hline * Statistical significant $P<0.05$. & & & & \\
\hline
\end{tabular}




\section{Discussion}

As mentioned, the incidence of BPH increases with age, and $\mathrm{BPH}$ is the most common cause of LUTS in elderly men. Among BPH patients with pre-existing comorbidities, quality of life is further impaired. Among elderly men, the co-occurrence of inguinal hernia and LUTS due to BPH is very likely, given that the prevalence of both conditions increases with age.

LUTS can have a negative effect on quality of life in BPH patients. IPSS, prostate volume, detrusor resistance index, intravesical prostatic protrusion, capillary artery resistive index, intraprostatic pressure, post-void residual, uroflowmetry, bladder wall thickness, and pressure flow assessments are commonly used to evaluate patients with LUTS due to BPH (5). In the present study, these methods were used to evaluate patients with $\mathrm{BPH}$, and those with and without inguinal hernia were compared. $\mathrm{BPH}$ and inguinal hernia are both associated with aging. An earlier study reports that patients with inguinal hernia tend to have higher IPSSs than patients without it; the difference is thought to be due to an increase in intraabdominal pressure in patients with obstructed voiding dysfunction, which can then lead to susceptibility to inguinal hernia formation (4). In the present study, there is no significant difference in IPSSs between the BPH and $\mathrm{BPH}-\mathrm{IH}$ groups $(\mathrm{p}=0.348)$ (Figure 1$)$, or in those between the BPH-IH subgroups A and B $(p=0.104)$.

Uroflowmetry is commonly used to evaluate the strength of urinary flow in men with LUTS. The maximum flow rate is the best indicator among all parameters that can be analyzed via uroflowmetry. It is important in diagnosing BPH, estimating the severity of obstruction, and evaluating treatment; however, to date, only a weak correlation has been reported between the maximum flow rate and symptom scores. In the present study, there was no significant difference in uroflowmetry parameters between the $\mathrm{BPH}$ and $\mathrm{BPH}-\mathrm{IH}$ groups $(\mathrm{p}=0.175)$.

\section{Figure 1.}

Boxplot of IPSS score in each group.

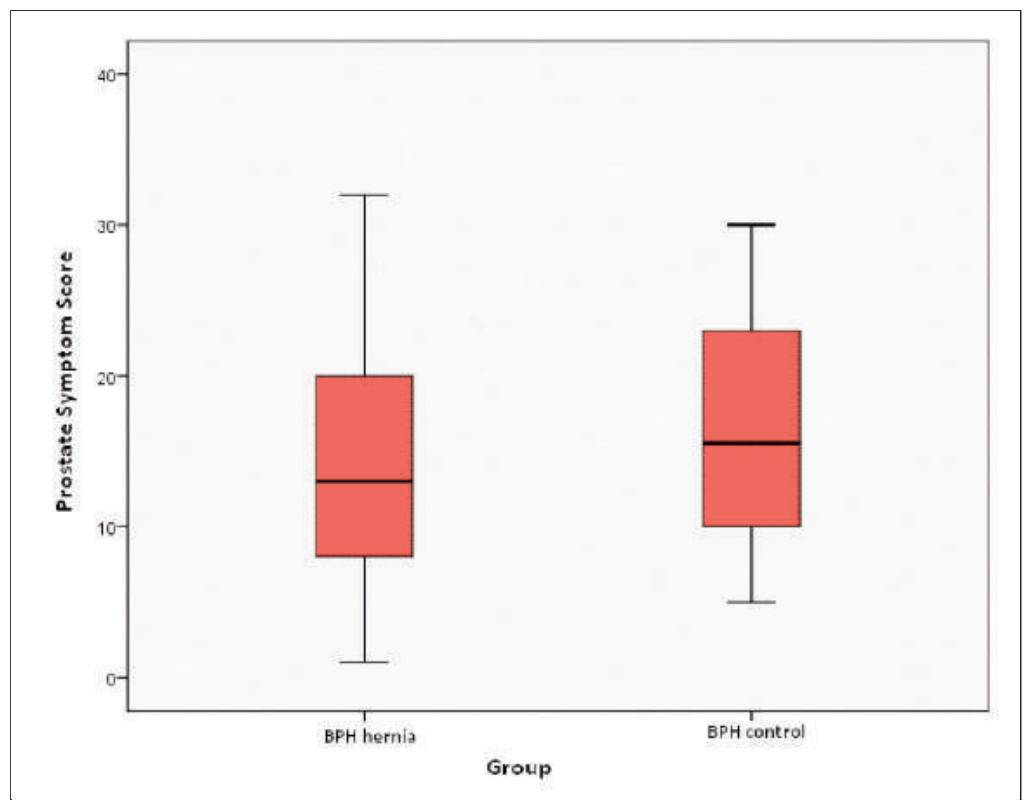

Ludwig et al. (6) report that 33\% of patients scheduled to undergo radical prostatectomy have concomitant inguinal hernia. Additionally, another study that investigated the incidence of inguinal hernia following prostate surgery, reports that the incidence rate of inguinal hernia after radical prostatectomy, open prostatectomy, and transurethral resection of the prostate (TUR-P) was $23.9 \%, 18.9 \%$, and $2 \%$, respectively. It is also reported that inguinal hernia develops during the first two years post surgery in $91.9 \%$ of patients that have undergone radical retropubic prostatectomy, and in $83.3 \%$ of patients that have undergone open prostatectomy (7).

Sanchez-Ortiz et al. (8) report that patients with an IPSS $>15$ before radical retropubic prostatectomy should be evaluated for additional surgical hernia repair. Irrespective of prostate size, patients with preoperative lower urinary tract dysfunction were reported to have a five-fold greater risk of undergoing an inguinal hernia repair procedure during radical prostatectomy. These two conditions are related to aging and commonly coexist. It has been reported that $11-30 \%$ of patients with LUTS who undergo a surgical procedure to repair inguinal hernia will develop urinary retention after surgery and require a urological intervention. General surgeons and urologists should be aware of this coexistence and inform patients about possible outcomes (4).

The general consensus is that these two surgeries should be performed simultaneously. Bawa et al. (9) report that there is no increase in duration of surgery or postoperative hospitalization among patients who undergo simultaneous inguinal hernia repair and TUR prostatectomy. In the present study, there was no significant difference in the prevalence of mild, moderate, or severe LUTS between the BPH and BPH-IH groups $(\mathrm{p}=0.348)$. Additionally, when patients in the $\mathrm{BPH}-\mathrm{IH}$ group were divided into subgroups A and B (direct and indirect inguinal hernia, respectively), the prevalence of mild, moderate, and severe LUTS did not differ significantly between the subgroups $(\mathrm{p}=$ 0.763).

\section{Conclusion}

In the present study, IPSS, uroflowmetry, and prostate volume were evaluated in $\mathrm{BPH}$ patients.

Our hypothesis was that patients who have high IPSSs on account of voiding dysfunction would have a higher IPSSs in the presence of an inguinal hernia; our results, however, do not support this hypothesis. This unexpected outcome may have derived from the small study population. Further larger-scale studies are needed to more clearly discern whether high IPSSs are associated with inguinal hernia.

\section{REFERENCES}

1. Berry SJ, Coffey DS, Walsh PC, et al. The development of human benign prostatic hyperplasia with age. J Urol 1984; 132:474-479. 
2. Wagner JP, Brunicardi FC, Amid PK, et al. Schwartz's Principles of Surgery, Tenth Edition. New York: McGraw-Hill Education. 2015; p 1495.

3. Rutkow IM. A selective history of groin hernia surgery in the early 19 th century. The anatomic atlases of Astley Cooper, Franz Hesselbach, Antonio Scarpa, and Jules-Germain Cloquet. Surg Clin North Am. 1998; 78:921.

4. Reis RB, Rodrigues Neto AA, Reis LO, et al. Correlation between the presence of inguinal hernia and the intensity of lower urinary tract symptoms. Acta Cir Bras. 2011; 26(Suppl 2):125-128.

5. Çakıroglu B, Sinanoglu O, Hazar AI, et al. Infravesical obstruction index in patients with benign prostatic hyperplasia is the best? JAREM. 2012; 2:109-112.

6. Ludwig WW, Sopko NA, Azoury SC, et al. Inguinal hernia repair during extraperitoneal robot-assisted laparoscopic radical prostatectomy. J Endourol. 2016; 30:208.

7. Sekita N, Suzuki H, Kamijima S, et al. Incidence of inguinal hernia after prostate surgery: open radical retropubic prostatectomy versus open simple prostatectomy versus transurethral resection of the prostate. Int J Urol. 2009; 16:110-113.

8. Sanchez-Ortiz RF, Andrade-Geigel C, Lopez-Huertas H, et al. Preoperative International Prostate Symptom Score predictive of inguinal hernia in patients undergoing robotic prostatectomy. J Urol. 2016; 195:1744.

9) Bawa AS, Batra RK, Singh R. Management of inguinal hernia with benign prostatic hyperplasia: simultaneous inguinal hernioplasty with transurethral resection of prostate. Int Urol Nephrol. 2003; 35:503-506.

\section{Correspondence}

Aykut Buğra Sentürk (Corresponding Author)

aykutbugra@gmail.com

Musa Ekici

Hitit University Corum Training and Research Hospital,

Urology Department, Turkey

Ibrahim Tayfun Sahiner

Hitit University Corum Training and Research Hospital, General Surgery Department, Turkey

Tuncay Tas

Istanbul Esenyurt University, Private Esencan Hospital,

Department of Urology, Turkey

Basri Cakiroglu

Hisar Intercontinental Hospital, Department of Urology, Turkey 